\title{
A mechanism for layer formation in stratified geophysical flows
}

\author{
J. L. Pelegrí and P. Sangrà \\ Departamento de Física, Facultad de Ciencias del Mar, Universidad de Las Palmas de Gran \\ Canaria, Canary Islands, Spain
}

\begin{abstract}
We discuss the possibility that steplike structures are formed in subcritical regions of vertically stratified shear flow. The mechanism we propose essentially consists of localized intense mixing in highly stratified and sheared flows, probably following frontogenesis. Its main assumption is that the vertical density flux increases monotonically with decreasing gradient Richardson numbers, which corresponds to enhanced stratification and/or diapycnal shear. This differs from Phillips' [1972] mechanism, which we argue may not apply to vertically stratified shear flow. An essential condition for the formation of constant density steps is the incorporation of a Langevin type equation which takes into account that turbulence must last for some finite characteristic time. We present numerical computations for the case of approximately constant diapycnal shear which lead to the formation of a staircase depth-density structure.
\end{abstract}

\section{The Phillips Mechanism}

Phillips [1972] suggested that small perturbations in an initially well stratified density profile will grow with time if a local increase in the vertical density gradient is accompanied by an even larger decrease of the (turbulent) vertical density diffusivity $K$. In this case, the vertical density flux will become smaller (larger) with increasing (decreasing) stratification, leading to vertical density divergence (convergence) where the density perturbation is negative (positive). The density perturbations will turn into progressively better defined steps, until the turbulent density flux becomes equal in the well-stratified and well-mixed portions of the staircase. He further parameterized $K$ in terms of some local Richardson number, to show that the density layers will form when the turbulent density diffusivity is a large enough inverse function of this local Richardson number.

Posmentier [1977] examined the stability of the same vertically diffusive density equation used by Phillips [1972] (but with salinity rather than density):

$$
\frac{\partial \rho}{\partial t}=-\frac{\partial F}{\partial z}=-\frac{\partial F}{\partial I} \frac{\partial^{2} \rho}{\partial z^{2}}
$$

where $\rho$ is density, $I=\partial \rho / \partial z$ is the local vertical density gradient, and $F \equiv \overline{w^{\prime} \rho^{\prime}}$ is the vertical component of the turbulent density flux vector $\vec{F}$. (Throughout the paper we will maintain the usual decomposition of instantaneous values as equal to ensemble mean plus

Copyright 1998 by the American Geophysical Union.

Paper number 98JC01627.

0148-0227/98/98JC-01627\$09.00 fluctuating values, and we will follow the notation that primed dependent variables refer to fluctuating quantities while unprimed dependent variables refer to ensemble mean quantities.) Posmentier pointed out that the solutions of this equation are unstable if $\partial F / \partial I$ is positive, and suggested that this is the case when stratification is larger than a certain critical value. He did some numerical calculations using a parameterization for the vertical density diffusivity of the type proposed by Munk and Anderson [1948]: $K=K_{0}(1+\beta R i)^{-n}$, where $K_{0}$ is the maximum $K$ value corresponding to vertical neutral conditions and $\beta, n$ are positive constants. In this expression, $R i$ is the local gradient Richardson number, defined as

$$
R i=\frac{-g\left(\frac{\partial \rho}{\partial z}\right)}{\rho\left(\frac{\partial v}{\partial z}\right)^{2}},
$$

where $v$ is the speed of the horizontal velocity, $\partial v / \partial z$ is the vertical shear, and $g$ is the gravity acceleration (the minus sign in the definition assures $R i$ to be positive for stable stratification). The numerical solution was able to reproduce the formation of a depth-density staircase structure. (Hereafter, whenever we use the symbol $R i$, we will refer to this local gradient Richardson number, while other possible definitions for the Richardson number will be adequately identified.)

Barenblatt et al. [1993] have extended Phillips' mechanism by allowing a finite time for the turbulence to adjust to the varying vertical density gradient. They specified this time delay through a linear expansion for the eddy diffusivity, which was then incorporated into the temperature vertical diffusion equation. They analyzed this equation and showed that the problem is mathematically well posed and the solution unique, and 
further calculated some numerical solutions which show the formation of staircase type structures. Kranenburg [1996] has explored the interaction of the mean buoyancy and velocity fields in vertical diffusivity equations for both variables. He considered the slowness in the turbulence temporal adjustment by assuming that the vertical eddy diffusivity must evolve forced through a Prandtl-Kolmogorov type source term. He did an stability analysis of the equations (using generalized MunkAnderson density and momentum diffusivity coefficients specified in terms of $R i$ ) and obtained rather restrictive conditions on the shape of both the vertical density flux and vertical momentum flux for the development of instabilities. He further showed that under these conditions the numerical solution of the density, momentum, and eddy diffusivity equations leads to the formation of evolving staircase type structures.

The key assumption for Phillips' mechanism is the dependence of $F$ on density stratification. This mechanism requires $F$ to be small both for well-mixed and highly stratified conditions, with a maximum at some critical stratification value. The physical justification is that for low stratification $\rho^{\prime}$ decreases while for high stratification $w^{\prime}$ is inhibited; additionally, under high stratification the correlation $\overline{\rho^{\prime} w^{\prime}}$ is reduced as the nature of the flow changes, with internal wave motions becoming more dominant [Ivey and Imberger, 1991]. Linden $[1979,1980]$ reviewed and did many laboratory experiments on grid-generated mixing across density interfaces and found that in all of them, independent of the stirring rate, the dependence was similar to that required by Phillips' mechanism. Linden [1980] combined the experimental data to show a similar dependence of the flux Richardson number (defined as the ratio of change in potential energy to the loss of turbulent kinetic energy) as a function of a local Richardson number expressed using some characteristic turbulent velocity scale. Ivey and Imberger [1991] have also found a parallel relation using data from grid-generated mixing experiments in stratified water and wind tunnels.

Ruddick et al. [1989] and Park et al. [1994] have recently presented laboratory experiments with mixing induced by stirring with arrays of vertical rods, leading to the formation of density steps. Park et al. [1994] defined an overall Richardson number depending on the initial stratification and on the speed of the rod. Their experiments clearly show that there is a critical value for this Richardson number (which depends on the Reynolds number), above which instabilities develop and turn into a set of temporally evolving and merging steps. They used a local Richardson number defined in terms of the step thickness and the speed of the rod. They found that the density flux decreased as this local Richardson number increased between 1 and 10 , beyond which it became uniform, but they could not accurately confirm that the density flux decreased for values below 1 . They further observed that the steps reached an equilibrium state which corresponded to a uniform density flux in the layered region.

These theoretical and experimental works appear to confirm the formation of a staircase type structure through a Phillips type mechanism. The similarity in the evolution and merging of steps seen in both Park et al.'s [1994] experiments and Barenblatt et al.'s [1993] and Kranenburg's [1996] numerical calculations is striking and rather convincing. One aspect that remains unclear, we believe, is the identification of the forcing mechanism that leads to the formation of the fine structure.

\section{What Is the Role of Vertical Shear?}

In the experiments reported by Ruddick et al. [1989] and Park et al. [1994] it is clear that turbulence is the result of the rods' movement, but we may wonder to what physical mechanism this corresponds in nature. In particular, it is hard to believe that this (external) source of turbulence corresponds to Kelvin-Helmholtz type instabilities, which develop in vertically stratified shear flow [Turner, 1986; Thorpe, 1987]. Hence it seems difficult to justify that the growth of the (experimentally and numerically) observed instabilities may have any dependence on $R i$, which is defined in terms of the local vertical shear.

The theoretical assumptions used by Phillips [1972] and Posmentier [1977] actually do not rely on any specific dependence of $F$ on $R i$ (although Posmentier [1977] did his numerical calculations using $R i$ calculated from a diagnostic depth dependence of the velocity profile). Instead they rely on the dependence of the vertical density diffusivity on a properly defined Richardson number, which is a function of the local vertical density gradient. To illustrate this, let us temporarily take a constant vertical shear, such that $R i=R i(I)$. In this case, equation (4) of Phillips [1972] may be differentiated to give

$$
\begin{aligned}
-\frac{\partial \overline{b^{\prime} w^{\prime}}}{\partial z} & =v_{*}^{2} V^{\prime} \frac{\partial\left[R i^{1 / 2} G(R i)\right]}{\partial R i} \frac{\partial R i}{\partial z} \\
& \propto-\frac{\partial g(I)}{\partial I} \frac{\partial^{2} \rho}{\partial z^{2}}
\end{aligned}
$$

where $\overline{b^{\prime} w^{\prime}}$ is the vertical buoyancy flux (proportional to the vertical density flux $F$ ), $v_{*}$ is an externally imposed characteristic velocity, $V^{\prime}=\partial v / \partial z$ is the (constant) vertical shear, and $g, G$ are undetermined functions of $I$ which satisfy $R i^{1 / 2} G(R i) \propto I^{1 / 2} G(I) \equiv g(I)$; note that the change in sign takes place because $R i$ is always taken as a positive value.

The same argument used in equation (1) allows us now to conclude that Phillips mechanism is really independent of the amount of vertical shear, i.e., it may happen for any $V^{\prime}$ different from zero. If $V^{\prime}>0$, density perturbations are unstable when $\partial\left(R i^{1 / 2} G\right) / \partial R i<0$ (Phillips' statement) or $\partial g / \partial I>0$ (Posmentier's statement); the opposite occurs for $V^{\prime}<0$. Of course, $V^{\prime}$ may not be constant in $z$ but the condition for instability does not depend on this. It may be clarifying that if we were to try the case of constant density stratification (constant $I$ ), then the instability condition could not be 
obtained. We may conclude that Phillips' mechanism is not caused by turbulence induced through vertical shear and that any analysis of this mechanism is inconsistent both with the specification of the vertical density diffusivity $K$ in terms of a local gradient $R i$ and with the utilization of a Munk-Anderson type of parameterization (which is supposed to apply only for stratified shear flow).

The above discussion is, of course, an oversimplification of the problem because it assumes that the vertical density flux will only depend on the density stratification, but it is illustrative of the sort of formal difficulties encountered if the density flux is left to depend on $R i$. This density flux should actually depend on the local vertical density gradient, on the rate of dissipation of turbulent kinetic energy $\epsilon$, on the kinematic viscosity $\nu$, and on the size $D$ and velocity $V$ of the eddies (proportional to the diameter and velocity of the rod). The numerical and experimental evidence supporting Phillips' mechanism suggests that the dependence of the vertical density flux on the local stratification $K(I)$ should lead to a $F(I)$ type of dependence with a single maximum, as required by Phillips mechanism. The main difficulty in assessing this dependence, however, is due to the fact that most results in the literature are in terms of an overall density stratification [Linden, 1979,1980; Ivey and Imberger, 1991].

Let us now consider the behavior of the vertical density flux $F$ for shear-induced instabilities. In this case the parameterizations employed for the vertical density diffusivity $K$ are frequently of the Munk-Anderson type; for reviews and applications, see Pacanowsk and Philander [1981], Turner [1986], Fernando [1991], and Pelegri and Csanady [1994]. Another type of parameterization corresponds to Peters et al. [1988], who obtained a two-regime functional form $K=K(R i)$ for the equatorial undercurrent, with a Munk-Anderson type expression for $R i \geq 0.4$ and an expression of the form $K \sim R i^{-9}$ for lower $R i$ values (Figure 1 ). $F$ is usually approximated in terms of $K$ as follows [e.g., Posmentier, 1977; Csanady, 1990; McDougall and You, 1990; Pelegri and Csanady, 1994]:

$$
F=-K \frac{\partial \rho}{\partial z}=-\frac{K}{J},
$$

where $J=1 / I=\partial z / \partial \rho$, or the Jacobian of the transformation from the vertical $(x, y, z)$ to the isopycnic $(x, y, \rho)$ reference system. An alternative expression for $F$ is obtained introducing equation (2) into the first equality of equation (4)

$$
F=\frac{\rho R i K}{g}\left(\frac{\partial v}{\partial z}\right)^{2}
$$

This suggests that with a Munk-Anderson type of parameterization, $F$ may have a maximum value at some low critical $R i$ value. However, if we accept Peters et al.'s [1988] dependence for low $R i$ values $\left(K \sim R i^{-9}\right)$, then $F$ probably increases continuously with decreasing Ri. Peters et al.'s [1988] high inverse dependence of $K$

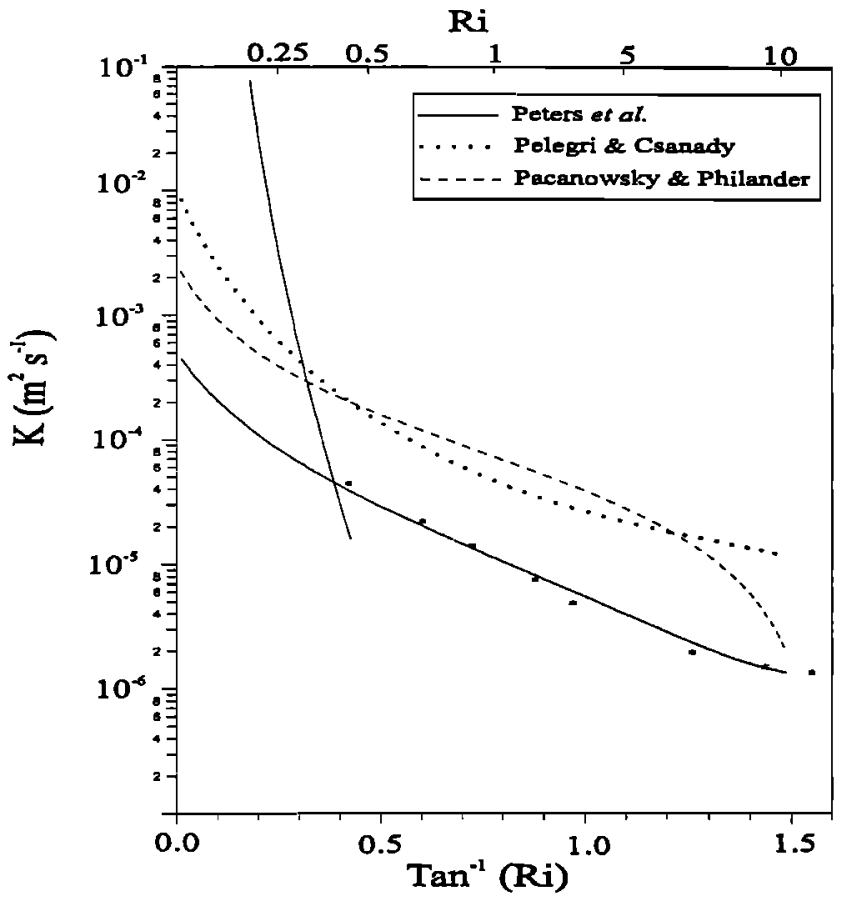

Figure 1. Vertical density diffusivity as a function of the local gradient Richardson number according to the parameterizations of Pacanowski and Philander [1981], Peters et al. [1988], and Pelegrí and Csanady [1994]. We have adapted a curve to the Peters et al. [1988] measurements (dots) for the $R i \geq 0.4$ region.

for low $R i$ values is very attractive because it conforms to a near-catastrophic shear-mixing criterion, with mixing taking place below a critical $R i$ value, in agreement with theoretical energy arguments [Miles, 1961; Howard, 1961; Pollard et al., 1973]. However, in both cases the exact behavior depends on how $\partial v / \partial z$ changes with decreasing $R i$.

For the particular case of constant $\partial v / \partial z$, we may define

$$
\left.F_{z} \equiv F\right|_{\frac{\partial v}{\partial z}}
$$

and use equation (5) to evaluate $\partial F_{z} / \partial R i=0$ for any parameterization of $K$, in order to find out if there is a value of $R i$ for which $F$ is a maximum. The utilization of a Munk-Anderson relation $K=K_{0}(1+\beta R i)^{-n}$ gives a vertical density flux with a maximum at $R i=$ $[\beta(n-1)]^{-1} ;$ for example, for $n=3 / 2$ and $\beta=10$ [Pelegri and Csanady, 1994], $F_{z}$ has a maximum at $R i=0.2$. If we use Peters et al.'s [1988] parameterization, however, we obtain no maximum, meaning that $F_{z}$ increases monotonically with decreasing $R i$. Figure 2a illustrates these results using both the Pelegri and Csanady [1994] and the Peters et al. [1988] expressions. For these calculations, we have used $\partial v / \partial z=10^{-2} \mathrm{~s}^{-1}$, of the same order as the maximum values reported by Bane et al. [1981] for the Gulf Stream.

Under the condition of constant vertical shear the Munk-Anderson parameterization (provided that $n>$ 1) results in a $F(R i)$ dependence which resembles the shape required for the Phillips mechanism. This pro- 

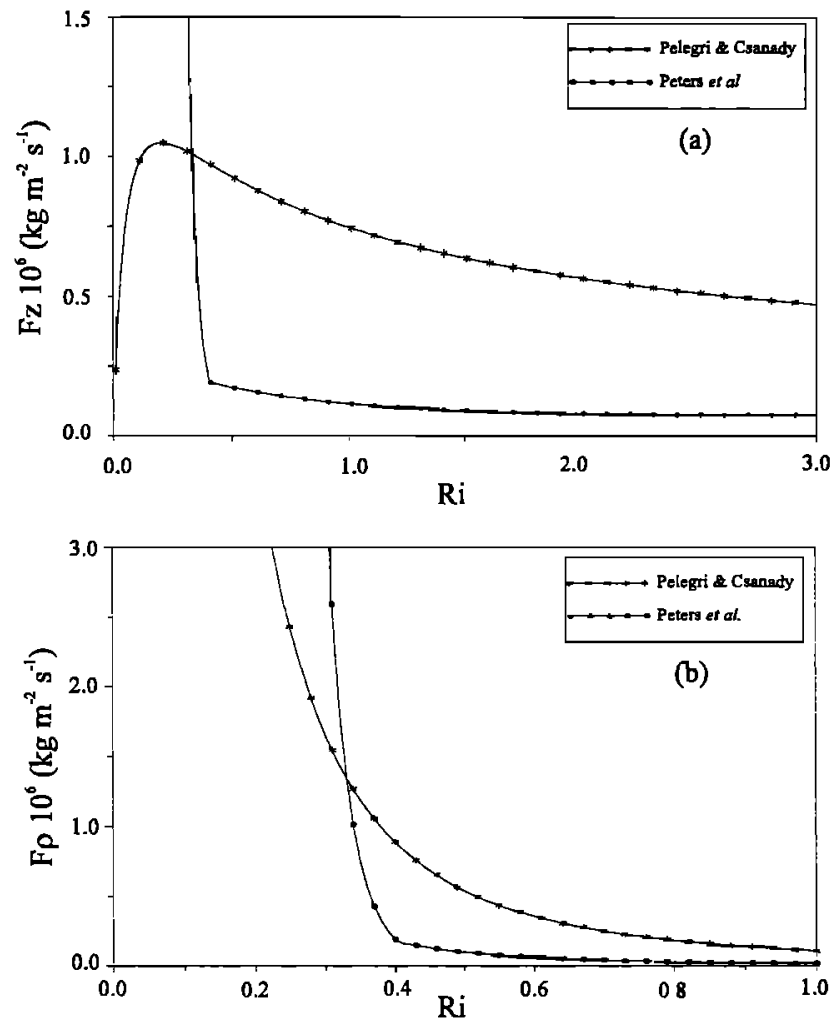

Figure 2. Vertical density flux as a function of the local gradient Richardson number for the cases of (a) constant vertical shear $\left(F \equiv F_{z}\right)$ and (b) constant diapycnal shear $\left(F \equiv F_{\rho}\right)$. The distributions are shown for both the Peters et al. [1988] and the Pelegrí and Csanady's [1994] $K(R i)$ parameterizations.

duces a factual justification (based on the facts but not on reasoning) for relating this mechanism to shearinduced instabilities. An adequate knowledge of the exact dependence $K(I, \epsilon, \nu, V, D)$, however, is very important in order to specify the instability conditions necessary for Phillips' mechanism to develop. In particular, this knowledge is critical when taking into account the interaction between the density and velocity fields. The inadequacy of a Munk-Anderson type of $K^{\prime}$ parameterization is probably the reason for the very restrictive instability conditions found by Kranenburg [1996].

A common discourse is that low- $R i$ values imply low vertical density gradients, which in turn, are responsible for very small density fluctuations and a decrease in $F$ [Posmentier, 1977; Ruddick et al., 1989; Park et al., 1994; Kranenburg, 1996]. This is a sensible argument for a Richardson number defined in terms of a (constant) characteristic turbulent velocity, which is related to the speed of the regularly moving rods. For shear-induced instabilities, however, we find no physical reason to support the assumption that $F$ has to decrease when $R i$ becomes small: these small values are only indicative of a very unstable situation, prior to very active mixing.

It must be emphasized that our purpose is not to oppose Phillips' mechanism but to warn that its origin has probably nothing to do with shear-induced mixing and to point out the necessity of expressing the vertical density flux in terms of some properly defined local Richardson number (instead of $R i$ ). This necessity has probably been appreciated by other authors such as Park et al. [1994], who always refer to a "suitably defined Richardson number" rather than to $R i$, but it is fair to say that the differences have not yet been properly pointed out. In the next section we will further illustrate the role of shear by considering the problem in isopycnic coordinates. This will naturally lead to an alternative mechanism for the formation of steps in vertically stratified flow, which operates in a completely opposite fashion and has its origin on instabilities induced by vertical shear.

\section{An Alternative View}

There is some uncertainty on how to apply the definition for $R i$ (equation (2)) to adjacent layers in a staircase type of stratification. In this case we have constant density layers separated by a stratified region, both usually of similar vertical thickness [Pelegrí and Csanady, 1994], and we may wonder which is the most appropriate vertical scale $\delta z$ to calculate the change in velocity $\delta v$ : the thickness of the whole region (both layers and interface) or the thickness of the stratified region (interface)? The answer, however, is immediate if we consider the problem in isopycnic coordinates because both constant density layers reduce to one single $\rho$ coordinate in the isopycnic coordinate system, each of them having an approximate constant velocity value. This practical advantage comes along with a powerful argument for using isopycnic coordinates, which was long ago clearly stated by Rossby $[1936$, p. 5$]$ : “... water, because of its stratification, has a cellular structure, each cell being separated through approximately horizontal surfaces of discontinuity from the cells above and below. Each boundary surface would then act as a 'false' bottom and each cell would have a practically independent circulation" (see also Csanady [1989] and Csanady and Pelegri [1995]).

The above reasoning suggests that the natural representation for $R i$ is using isopycnic coordinates [Pelegri and Csanady, 1994]:

$$
R i=\frac{-g J}{\rho\left(\frac{\partial v}{\partial \rho}\right)^{2}} .
$$

In this case the vertical density flux becomes

$$
F=\frac{g K}{\rho R i\left(\frac{\partial v}{\partial \rho}\right)^{2}},
$$

and we may again ask ourselves what is the behavior of $F$ as a function of $R i$. The key factor now is not the vertical shear, but the diapycnal shear, $\partial v / \partial \rho$, i.e., the dependence of this quantity with $R i$. The simplest possibility is to assume $\partial v / \partial \rho$ constant, as we did before for $\partial v / \partial z$. In this case we define

$$
\left.F_{\rho} \equiv F\right|_{\frac{\partial v}{\partial \rho}}
$$


and we can use equation (7) to evaluate $\partial F_{\rho} / \partial R i=0$ for any particular parameterization of $K$. This calculation shows that $F_{\rho}$ has a maximum neither for the Munk-Anderson nor for the Peters et al. [1988] parameterizations, in both cases $F_{\rho}$ monotonically increases for decreasing $R i$. Figure $2 \mathrm{~b}$ illustrates these results using both the Pelegri and Csanady [1994] and the Peters et al. [1988] expressions. For these calculations we have used $\partial v / \partial \rho=2.5 \mathrm{~m}^{4} \mathrm{~kg}^{-1} \mathrm{~s}^{-1}$, of the same order as the maximum values reported by Pelegri and Csanady [1994] for the Gulf Stream. The curve corresponding to the Pelegri and Csanady [1994] parameterization (of the Munk-Anderson type) is strikingly different to its counterpart in Figure 2a.

Equations (2) and (6) are the expressions for $R i$ in vertical and isopycnic coordinates, respectively. At first sight, they may suggest very different situations: equation (2) shows that $R i$ increases with stratification (larger $\partial \rho / \partial z$ ), while equation (6) shows that $R i$ decreases with stratification (smaller $J=\partial z / \partial \rho$ values). The solution of this apparent paradox rests on the velocity profile, the diapycnal and vertical shears being related through the Jacobian, i.e., $\partial v / \partial \rho=J \partial v / \partial z$. For staircase type conditions, the conceptual idea of independent motions along adjacent constant density layers suggests that the difference in velocity between adjacent isopycnals will usually be a much better conserved quantity than vertical shear, which probably has a strong dependence on the evolution of the interfaces. With this assumption, equation (7) shows that the vertical density flux will have a local maximum at the position of highest stratification (the minimum in $J$ ): this is a statically stable region but it turns out to be dynamically very unstable [Pelegri and Csanady, 1994].

In view of the lack of experimental or field data, it is clear that the assumption of constant diapycnal shear cannot be generalized, but it is attractive under frontogenetical conditions. Consider, for example, the very simple case where the isopycnals are vertically and horizontally compressed with time but maintain their slope. Recalling the thermal wind equation in isopycnic coordinates $(\rho f \partial v / \partial \rho=g \partial z / \partial x)$, we may appreciate that under the geostrophic approximation a constant diapycnal shear would be appropriate. Under the same approximation, however, the increased horizontal density gradient would lead to an increase in vertical shear. Consider now a slightly more complex situation, with the isopycnals getting both compressed and tilted with time. This would cause a decrease in the Jacobian and an increase in both the diapycnal and vertical shears. In these circumstances the diapycnal shear is not constant, but it is probably more realistic to let $J$ decrease and take a constant $\partial v / \partial \rho$ (leading to a decrease in $R i$ through equation 6 ) rather than allowing $J$ to decrease and taking a constant $\partial v / \partial z$ (producing an increase in $R i$ according to equation (2)).

Following the above discussion, we are now ready to discuss an alternative process that leads to the formation of a step of constant density, i.e., to the partition of the single minimum in $J$ into two adjacent minima

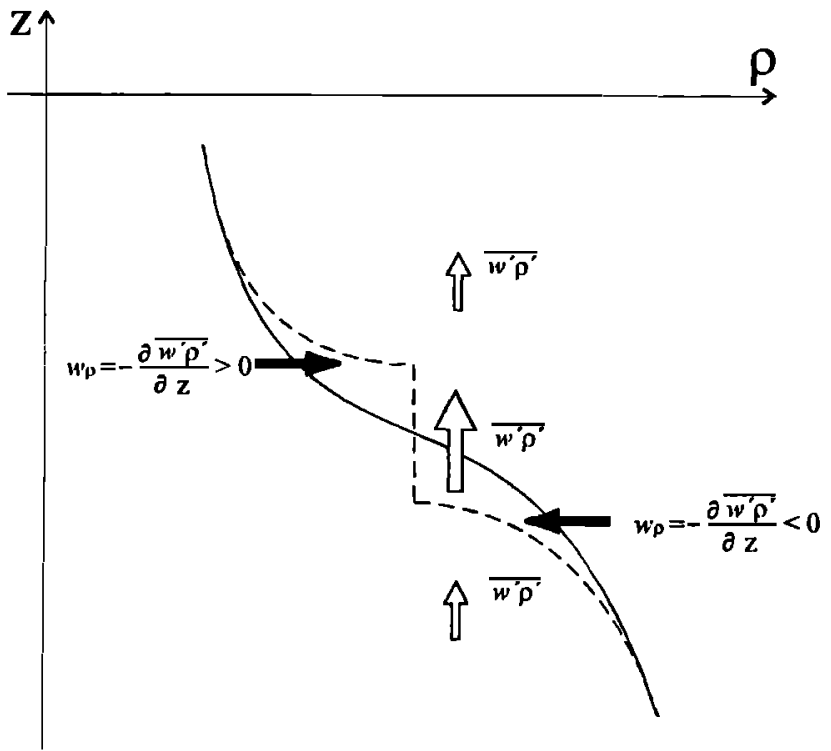

Figure 3. Schematic representation of density stratification before (solid line) and after mixing in the central region (dashed line). The arrows represent the situation previous to intense mixing: the white arrows represent the vertical density flux, and the black arrows represent the density tendency.

as illustrated in Figure 3. The density tendency, or material derivative of the density, is given by [Pelegri and Csanady, 1994]

$$
w_{\rho}=\frac{D \rho}{D t}=-\frac{\partial \overline{w^{\prime} \rho^{\prime}}}{\partial z},
$$

where $D \rho / D t=\partial / \partial t+u \partial / \partial x+v \partial / \partial y+w \partial / \partial z$ is the material derivative in $(x, y, z)$ coordinates. The solid line in Figure 3 represents the original density stratification, with a central highly stratified region which corresponds to the minimum absolute $J$ values. The upper part of the region of high stratification corresponds to vertical convergence of density flux (positive $w_{\rho}$ ), while its lower part corresponds to vertical divergence (negative $w_{\rho}$ ). This situation, if maintained for a finite time, will cause a density increase in the upper part and a density decrease in the lower portion (dotted line). This will naturally lead to a decrease of the stratification in the central region (the creation of a well-mixed region) and to an increase at both sides of this newly formed mixed region (the creation of interfaces). A similar argument could now apply to the new interfaces, which should be prone to undergo mixing and to split into new steps of a forming staircase.

Another way to visualize the above idea is through the mass conservation equation in isopycnic coordinates. Pelegri and Csanady [1994] showed that under the condition of dominant diapycnal convergence/diver gence $\partial w_{\rho} / \partial \rho$, i.e., neglecting epipycnal convergence/ divergence and horizontal Reynolds mass fluxes, the mass conservation equation reduces to

$$
\frac{\mathcal{D} j}{\mathcal{D} t} \simeq-j \frac{\partial w_{\rho}}{\partial \rho}
$$


where $j=J \rho$ is an index of separation between adjacent isopycnals and $\mathcal{D} / \mathcal{D} t=\partial / \partial t+u \partial / \partial x+v \partial / \partial y+$ $w_{\rho} \partial / \partial \rho$ is the material derivative in isopycnic coordinates. Applying this equation to the situation in Figure 3 , we can see that it gives $\mathcal{D} j / \mathcal{D} t<0$ for the central region, indicating that the absolute value of $J=j / \rho$ will increase with time, i.e., a decrease in the degree of stratification. Similarly, it may be appreciated that the stratification will increase with time in the upper and lower portions of the central region.

The creation of well-mixed regions following a localized maximum in the vertical density flux was already proposed by Pelegrí and Csanady [1994] in a simple cause-effect model. They hypothesized a distribution of the vertical density flux with a maximum on some isopycnal level and estimated the corresponding $J$ and $R i$ distributions, with the $R i$ distribution showing a minimum below the critical $R i$ value. Their assumption was that this condition, if maintained for a finite time, would cause the vertical density flux to be largely reduced in the subcritical region. They simulated the modified $\overline{w^{\prime} \rho^{\prime}}$ distribution and recalculated the $J$ distribution and the $z(\rho)$ profile, which showed the formation of one step. In their paper Pelegrí and Csanady [1994] proposed that mixing would be induced in shear unstable regions after frontogenesis, but they inaccurately mentioned that the physical process resembles Phillips' mechanism.

Figure 4 illustrates the instability processes for both the Phillips and the new mechanism, emphasizing their completely different kinematics. In Phillips' mechanism a perturbation that reduces the stratification of the depth-density profile will grow in time until it becomes a well-mixed region and adjacent well-stratified layers are formed (Figure 4a). In the proposed alternative mechanism a highly stratified region is unstable and mixes up, splitting into a well mixed region and two adjacent interfaces (Figure 4b).

The production of local oceanic finestructure through a mechanism based on localized shear-induced mixing in stratified regions was long ago endorsed by Woods [1968] and Woods and Wiley [1972], and discussed at some length by Turner [1973, pp. 104, 121, 325]. They argued that the passage of long internal waves along sharp interfaces can lead to local instabilities in well stratified regions, particularly at the crests and troughs of internal waves. Portions of the stratified region are then substituted by relatively well mixed fluid, which is then elongated horizontally by vertical shear and epipycnal spreading. Some oceanic observations on local mixing in stratified regions, apparently associated to crests and troughs of traveling internal waves, have also been documented by Woods [1968] and Woods and Wiley [1972].

The mechanism here proposed does not require the passage of internal waves and can operate on horizontal scales much larger than localized regions over crests or troughs of internal waves. It is based on the creation of subcritical regions during frontogenetical situations, either because of shrinking of isopycnal layers ( $J$ is reduced) or because of tilting of the isopycnals, followed
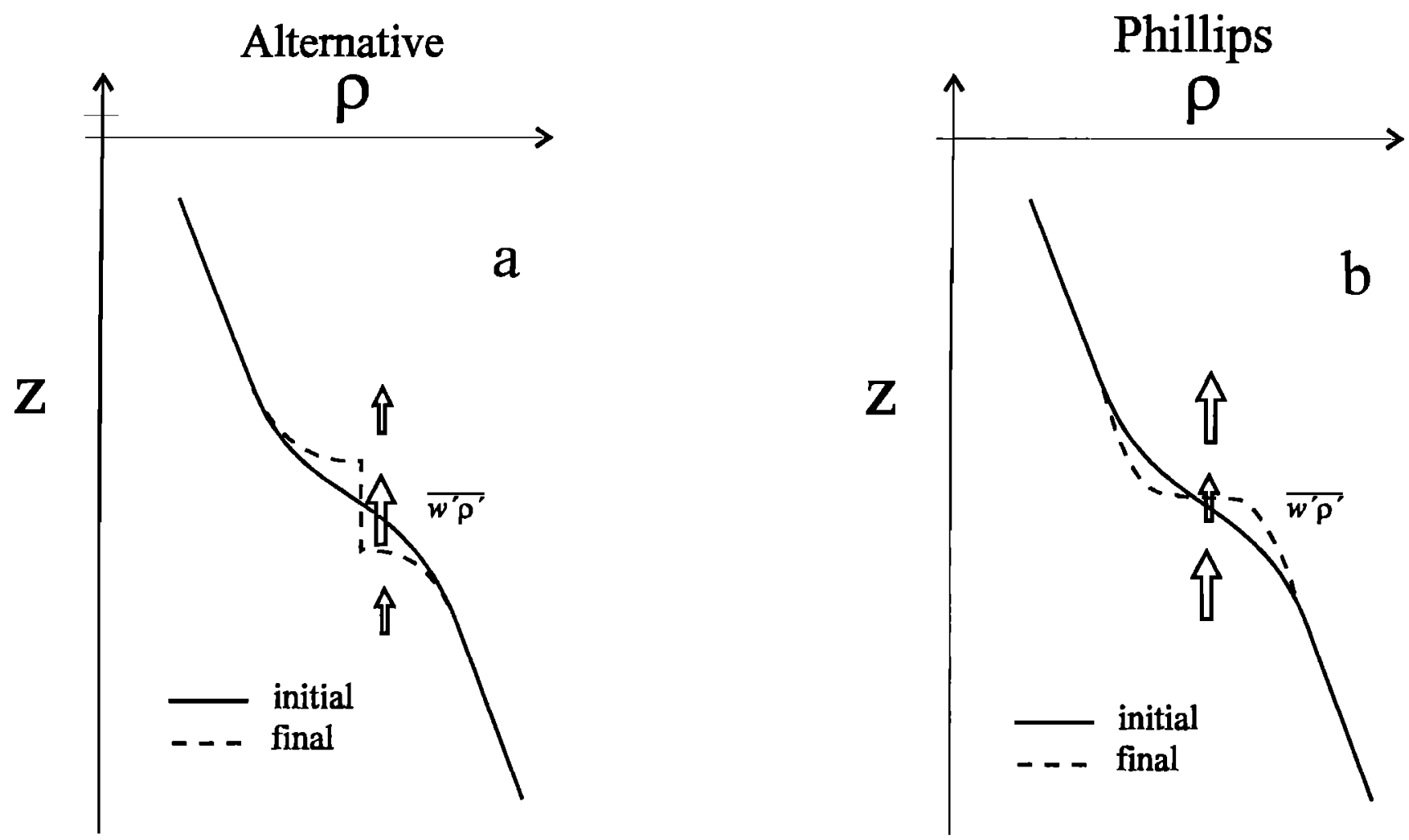

Figure 4. Schematic drawings to illustrate the kinematics of mixing for (b) Phillips' mechanism and (a) the proposed alternative mechanism. 
by instabilities of the Kelvin-Helmholtz type. The actual condition for the well stratified layers to become subcritical is derived from equation (6):

$$
J<\frac{\rho}{g}\left(\frac{\partial v}{\partial \rho}\right)^{2} R i_{c},
$$

where $R i_{c} \simeq 0.25$ is the theoretical critical value of $R i$ for mixing to develop.

Some laboratory evidence that supports this mechanism can be found in a preliminary experiment reported by Thorpe [1971]. In this experiment a long tank was completely filled with two fluids of different density and tilted for a short period, just enough for the interface to become unstable, and then returned to its horizontal position. Kelvin-Helmholtz instabilities developed at the interface, which became approximately well mixed, and two interfaces were formed at the edges of this central mixed region. Several related experiments have been recently performed, and some preliminary results appear to confirm the splitting of a thin interface into a well-mixed layer and adjacent interfaces [CisnerosAgurrre et al., 1997; Fernando, 1997].

Some interesting atmospheric data were long ago presented by Browning and Watkins [1970]. They used a high power radar and simultaneous radiosonde ascents to examine the evolution of regions of high static stability. Their observations showed the formation and evolution of Kelvin-Helmholtz billows in a highly stratified region, which lead to the splitting of this region into an approximately mixed layer and two adjacent interfaces. The oceanographic field evidence, however, is limited by the difficulties in repeating hydrographic cross sections in times shorter than the passage of frontal mesoscale features. Some indirect evidence, obtained through analyses of the distribution and fluxes of properties such as nutrients and potential vorticity, is consistent with the intermittent mixing associated with this mechanism [Pelegri and Csanady, 1991; Pelegrí et al., 1996].

The horizontal and vertical extension of the subcritical regions depend on the frontogenetical mechanism itself, whether it is a mesoscale or basin-wide feature. Those layers with high stratification during the initial stages of frontogenesis will be susceptible to convert into well-mixed regions of the staircase, while sharp interfaces will form at the adjacent layers. If frontogenesis continues, the adjacent risers will also mix up, each splitting into a new mixed layer and two new adjacent interfaces; eventually, diapycnal mixing may become so large that it can prevent further frontogenesis to continue. The vertical and temporal scales for the formation of the well-mixed regions will arise from the corresponding scales of the Kelvin-Helmholtz billows, which depend mainly on the vertical stratification. In the laboratory, for example, stratification is usually very large, and the height of the billows may be of the order of 0.01 $\mathrm{m}$, while their timescale may be of the order of $1 \mathrm{~s}$; in an oceanic frontal system, however, these scales could be 1-10 $\mathrm{m}$ and up to 1 hour.

In general, we may expect that the strongest oceanic frontogenetical processes will be intermittent and usu- ally associated with mesoscale features such as meanders in the Gulf Stream [e.g., Csanady, 1988; Lee and Csanady, 1994] or the coastal upwelling jet [e.g., Bane et al., 1981; Csanady, 1977, 1982], evolving vortices or rings [e.g., Miller and Evans, 1985; Arísteguı et al., 1994], or other types of frontal structures [e.g., Csanady, 1981; Simpson, 1981]. In all these cases the temporal scale of maximum frontogenetical conditions will last long enough (of the order of several hours or days) to guarantee that subcritical conditions are attained.

\section{Temporal Memory of Turbulence}

One point crucial to our previous analysis is the creation of a maximum density flux in the highly stratified region. It is not enough, however, to generate such an instantaneous maximum: for our mechanism to work it is fundamental that the density flux (with convergence and divergence regions above and below, respectively) is maintained at a high level for a finite time (Figure 3). Otherwise, the convergence and divergence of density toward the neighboring regions will reduce the original density gradient (increase the Jacobian), and the density flux will consequently decrease. The result will simply be upward mass diffusion and smoothing of the original strong density gradient. The temporal maintenance of such a high-density flux at the location of the minimum in $J$ has its physical justification on the fact that once turbulence is produced it will last for a characteristic finite time at a considerably high level. Even if the original unstable conditions disappear, turbulence will not immediately do so, i.e., once it is activated, it will decay at its own natural rate.

Barenblatt et al. [1993] and Kranenburg [1996] have pointed out the necessity of allowing a finite time adjustment for turbulence and have applied this idea to modify Phillips' mechanism. Such a time adjustment is not critical for their solution to work, although Barenblatt et al. [1993] have shown that it is necessary in order to make the problem mathematically well posed and the solution unique. Barenblatt et al. [1993] specified the time delay by using an elegant simple temporal expansion for the vertical density diffusivity. Kranenburg [1996] followed the same idea but justified the temporal evolution of the vertical density diffusivity through forcing by a Prandtl-Kolmogorov type source term.

In this work we essentially follow Barenblatt et al.'s [1993] approach to take account of the temporal memory of the turbulence field. This is done in a very simple manner, by approximating the vertical density diffusivity $K$ with the first term of a Taylor expansion:

$$
K(t)=K(t-\tau)+\left(\frac{\partial K}{\partial t}\right)_{(t-\tau)} \tau .
$$

This equation, which relates the vertical density diffusivity at time $t$ with those conditions that took place some time $\tau$ ago, immediately suggests equation (9) of Kranenburg [1996]:

$$
\frac{\partial K_{t}}{\partial t} \tau=-K_{t}+K_{f},
$$


where we have defined two different functions which correspond to the vertical density diffusivity evaluated at different times, $K_{f} \equiv K(t)$ and $K_{t} \equiv K(t-\tau)$. Equation (12) is Langevin's equation and has its appealing physical meaning of a slowly decaying field (the turbulence with its own characteristic temporal scale) which is continuously changing due to external forcing (the local stratification); for a nice discussion see Csanady [1973]. If there was no external forcing, the solution would decay exponentially as $\exp (-t / \tau)$, which shows that $\tau$ corresponds to the characteristic temporal scale of the turbulence.

All that is required now is to specify a value for $\tau$. Under the restoring force of gravity, perturbations have an angular velocity which is given by the buoyancy frequency $N$, defined in terms of the local vertical density gradient as $N^{2}=-g / j$ Hence it may be expected that the natural period for the vertical density flux will be of the order of $N^{-1}$. This agrees well with numerical and experimental results compiled by Gregg [1987], which show that the age of the energy-containing eddies will be somewhere between $0.1 N^{-1}$ and $N^{-1}$. More recent numerical calculations by Holt et al. [1992] also show that the vertical density flux remains large for times of the order of $N^{-1}$. According to these arguments, we have chosen $\tau=N^{-1}$ as the characteristic temporal scale of turbulence, to be used in the numerical calculations below. Because of the importance of local stratification in specifying the vertical density flux, we have decided to let $\tau$ vary vertically and temporally, depending on the instantaneous local vertical stratification. This has been preferred over the alternative of specifying $\tau$ in terms of the background initial stratification (as is done by Kranenburg [1996]).

\section{Numerical Procedure}

For dominant diapycnal mass transfer, the advective terms $u \partial j / \partial x+v \partial j / \partial y$ are relatively small and equation (9) may also be approximated as

$$
\frac{\partial j}{\partial t} \simeq-\frac{\partial\left(j w_{\rho}\right)}{\partial \rho}
$$

where we have incorporated the diapycnal advection of $j$ into the right-hand side. This equation simply states that changes in the distance between adjacent isopycnals are due to diapycnal convergence/divergence; it could have been derived from first principles by recalling that the mass flux (per unit area) through entrained water (water crossing an isopycnal with vertical speed $w_{e}$ ) is given by $\rho w_{e}=\rho J w_{\rho}=j w_{\rho}$.

Equation (12) provides the time evolution of the current density diffusivity $K_{t}$, which slowly changes as it decays and is forced by the eddy diffusivity $K_{f}$ calculated from the local density field. In order to solve it, we will use a functional form $K_{f}=K_{f}(R i)$ similar to that proposed by Peters et al. [1988], mainly because it conforms well to the catastrophic dependence that is expected for subcritical $R i$ numbers. For values of
$R i<0.33$, we will use exactly the same dependence as specified by Peters et al. [1988] $\left(K_{f} \propto R i^{-9.2}\right)$, while for the range $R i \geq 0.33$, we will use Pelegri and Csanady's [1994] parameterization (Figure 1 illustrates that both functions intersect at $R i=0.33$ ). A second possibility would be to use a Munk-Anderson parameterization for all $R i$ values. The exact $K_{f}$ dependence is not decisive, for constant diapycnal shear both the Peters et al. and the Munk-Anderson parameterizations result in $K_{f}$ monotonically increasing with decreasing stratification, but some important differences arise (see the discussion at the end of section 6).

Summarizing, we will solve equations (12) and (13) together with the following parameterizations for density tendency:

$$
w_{\rho}=\frac{\partial}{\partial z}\left(\frac{K_{t}}{J}\right)=\frac{1}{J} \frac{\partial}{\partial \rho}\left(\frac{K_{t}}{J}\right)
$$

and for the forcing vertical density diffusivity:

$$
K_{f}= \begin{cases}1.1 \times 10^{-8} R i^{-9.2} & , R i<0.33 \\ 2.6 \times 10^{-3}(1+10 R i)^{-3 / 2} & , R i \geq 0.33\end{cases}
$$

where $R i$ is given by equation (6). According to the discussion in last section, we will a priori specify a value for the diapycnal shear, with the main test case corresponding to constant diapycnal shear.

These equations are solved numerically in the $(t, \rho)$ space (forward in time and centered in $\rho$ ) to predict the temporal evolution of the vertical density diffusivity and Jacobian fields. The grid spacing is chosen as $\delta \rho=0.005 \mathrm{~kg} \mathrm{~m}^{-3}$ and $\delta t=0.1 \mathrm{~s}$. Equation (13) requires the specification of boundary conditions for $j$; these have been chosen as constant $j$ values, which imply that at the boundary isopycnals there is no diapycnal convergence/divergence. Once the Jacobian $J=j / \rho$ is known, we may immediately calculate the depth field $z$ through integration from $z=0$. Since we consider constant diapycnal shear, equation (15) shows that $\partial K_{f} / \partial \rho$ may be discontinuous at those density values where $R i=0.33$. This causes some small numerical instabilities, which are removed (at each time step) by applying Shapiro's running filter [Shapiro, 1970] three times to $K_{f}$.

The initial stratification corresponds to the depth linearly decreasing with density (from 0 to $-200 \mathrm{~m}$ in 0.5 $\mathrm{kg} \mathrm{m}^{-3}$ ), over which we superimpose a sinusoidal depth fluctuation ( $25 \mathrm{~m}$ fluctuation with wavelength $\lambda_{\rho}=0.5$ $\mathrm{kg} \mathrm{m}^{-3}$ ). The amplitude of the fluctuation is chosen such that its maximum slope is less than the background density slope. The diapycnal shear has been taken as $2.5 \mathrm{~m}^{4} \mathrm{~kg}^{-1} \mathrm{~s}^{-1}$, of the same order of magnitude as the maximum values reported by Pelegri and Csanady [1994]. Figure 5 shows the initial conditions specified for the $z(\rho)$ field, and the corresponding initial condition for the Jacobian $J$, the vertical density flux $\overline{w^{\prime} \rho^{\prime}}$, and the density tendency $w_{\rho}$. Hereafter, in all figures we use $\sigma \equiv \rho-1000$, with $\rho$ being the absolute value of density in MKS units. 

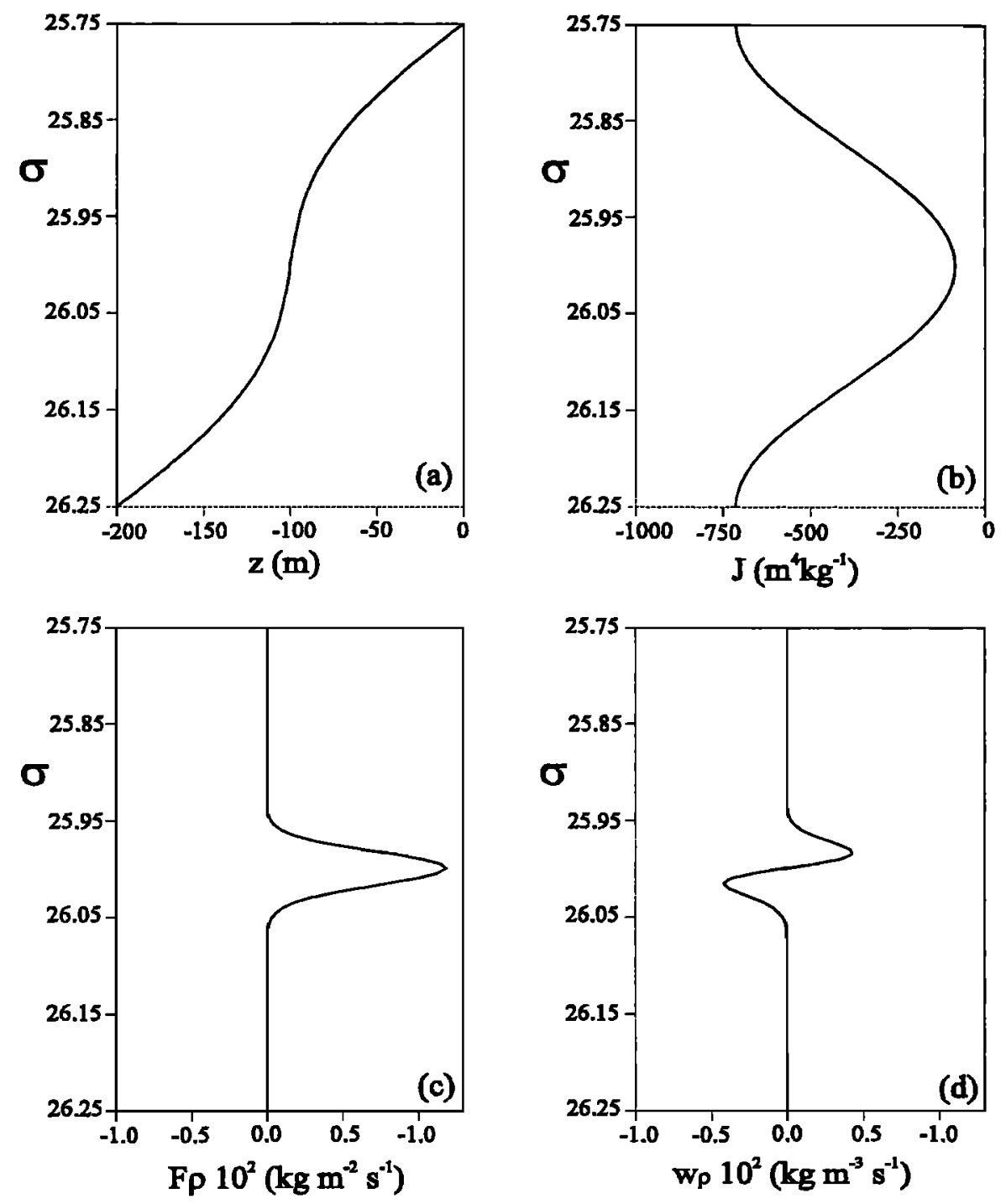

Figure 5. Initial conditions for the numerical calculations. (a) The density stratification is specified as the sum of a background linear stratification and a wave type depth-density perturbation. From the $z=z(\rho)$ field and using $\partial v / \partial \rho=2.5 \mathrm{~kg} \mathrm{~m}^{-3} \mathrm{~s}^{-1}$, we obtain the initial (b) Jacobian, (c) vertical density flux, and (d) density tendency.

\section{Results and Discussion}

Figure 6 shows the temporal evolution of the relevant variables for the purely diffusive case, which corresponds to $\tau=0$. In this case the system has no memory of turbulence: the maximum in the vertical density flux is responsible for the very rapid initial density convergence/divergence, but the vertical density flux decays simultaneously with the reduction of the density gradient, which inhibits the maintenance of the large density convergence/divergence necessary to produce the well-mixed region. The result is rather rapid density diffusion and the smoothing of the well-stratified region (Figures 6a and 6b), the vertical density flux (Figure $6 \mathrm{c}$ ), the density tendency (Figure 6d), the diffusion coefficient (Figure 6e; since $\tau=0$, this case corresponds to $K_{t}=K_{f}$ ), and the local gradient Richardson num- ber (Figure $6 \mathrm{f}$; since $\partial v / \partial \rho$ is constant, $R i$ parallels $J$, Figure $6 \mathrm{~b}$ ). Figure $6 \mathrm{f}$ illustrates that $R i$ remains low during a long period but it is almost constant over a large density range, implying that $K_{f}$ (Figure 6e) is rather smooth.

Figures 7 and 8 show the temporal evolution for the case where we choose $\tau=N^{-1}$, as discussed in section 4. The initial $\tau$ values, for example, range somewhere between $200 \mathrm{~s}$ and $100 \mathrm{~s}$ for those regions with minimum and maximum stratification, respectively. Figure 7 presents the evolution of the depth, Jacobian, vertical density flux, and density tendency at different times. The situation is quite different from the diffusive case $(\tau=0)$, and it shows how the initially well-stratified region turns into a well-mixed region (Figures $7 \mathrm{a}$ and $7 \mathrm{~b})$. This mixed region is actually produced by the slow decrease experienced in the vertical density flux and the 

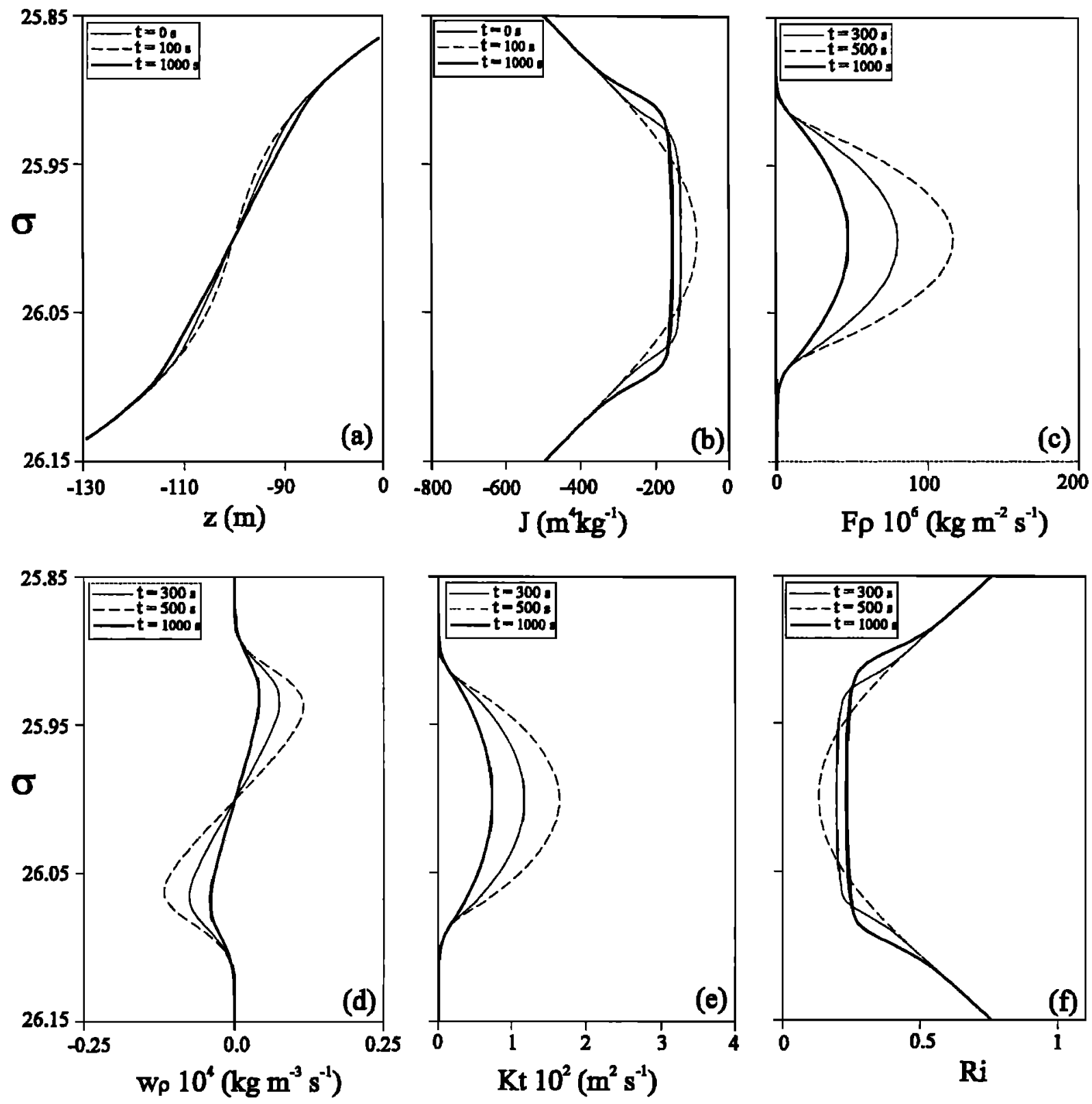

Figure 6. Case with $\tau=0$, showing the temporal evolution of the density distribution for (a) depth, (b) Jacobian, (c) vertical density flux, (d) density tendency, (e) actual density diffusivity, and (f) local gradient Richardson number.

density tendency (Figures $7 \mathrm{c}$ and $7 \mathrm{~d}$ ). Figure 8 clearly illustrates the different behavior of $K_{t}$ and $K_{f}$, showing how the forcing density diffusivity $K_{f}$ is rapidly reduced (Figure $8 \mathrm{~b}$ ) while the actual density diffusivity $K_{t}$ remains high much longer due to the temporal memory of turbulence (Figure 8a). Figure 8c shows the evolution of $R i$ (again paralleling the Jacobian): the initial minimum is rapidly replaced by a maximum with two adjacent minima. The maximum corresponds to the formation of the well mixed layer, while the minima indicate the locations prone to undergo future mixing.

Figures 9 and 10 correspond to the same case as before, with the difference that Shapiro's running filter is applied 30 times to $R i$ at each time step. Note that averaging $R i$ but not $J$ breaks the constant diapycnal shear assumption (equation (6)). The justification for doing so comes from the anisotropy associated with Kelvin-Helmholtz instabilities, which implies that they cannot take place at scales shorter than the Ozmidov scale [Miller and Evans, 1985]; by averaging only $R i$, we ponder this minimum vertical size of shear-induced turbulence. Several studies [Miller and Evans, 1985; Gregg, 1987] suggest that in moderately to highly stratified regions the Ozmidov scale will be about $2-3 \mathrm{~m}$. Taking $3 \mathrm{~m}$ and dividing by $100 \mathrm{~kg} \mathrm{~m}^{-3}$ (a characteristic value of the Jacobian in the well stratified regions) gives a corresponding density scale of $0.03 \mathrm{~kg} \mathrm{~m}^{-3}$. This shows that about six density levels in our model (re- 

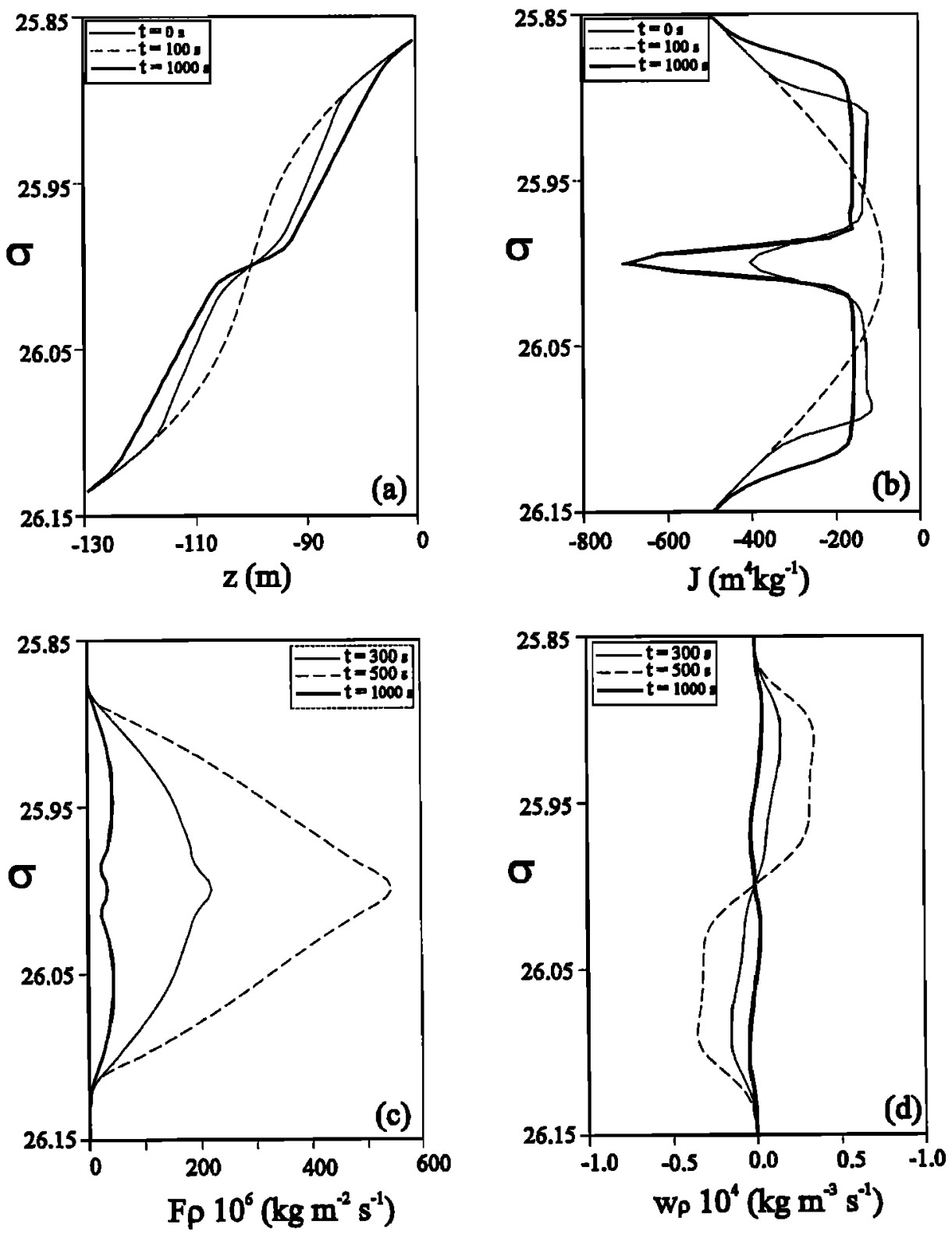

Figure 7. Case with $\tau=N^{-1}$, showing the temporal evolution of the density distribution for (a) depth, (b) Jacobian, (c) vertical density flux, and (d) density tendency.

call $\delta \rho=0.005 \mathrm{~kg} \mathrm{~m}^{-3}$ ) should be averaged to avoid contamination from small-scale turbulence; this is approximately attained with the above running filter. The vertical averaging is clear in the $R i$ distribution (Figure $10 \mathrm{c}$ ), which now shows substantial differences from the $J$ distribution (Figure $9 \mathrm{~b}$ ). The main difference with the previous calculations is that the maxima and minima in the different variables are better defined, which results in a clearer steplike structure: the central mixed layer and adjacent interfaces are sharper and additional steps of the staircase are also suggested (Figure 9a).

Several additional runs have been done (1) to examine the differences related to using either the MunkAnderson or the Peters et al. parameterizations, and (2) to assess the sensitivity to the constant diapycnal shear assumption. The results are not shown here for the sake of brevity, but they indicate that the MunkAnderson parameterization can also lead to the generation of mixed layers. The difficulty with this parameterization, however, is that the above initial conditions give values of $w_{\rho}$ which are several orders of magnitude too small for the mechanism to operate. In order to attain sufficiently large density tendency values this requires some extra forcing, either through a much larger diapycnal shear or a much smaller Jacobian. A low Jacobian, however, implies a too short turbulent temporal memory $\left(\tau=N^{-1}\right)$, so the mechanism operates only with unrealistically high diapycnal shear. The second conclusion arising from the test runs is that the assumption of constant diapycnal shear is not critical for the proposed mechanism to operate. This was actually anticipated by the numerical runs shown in Fig- 

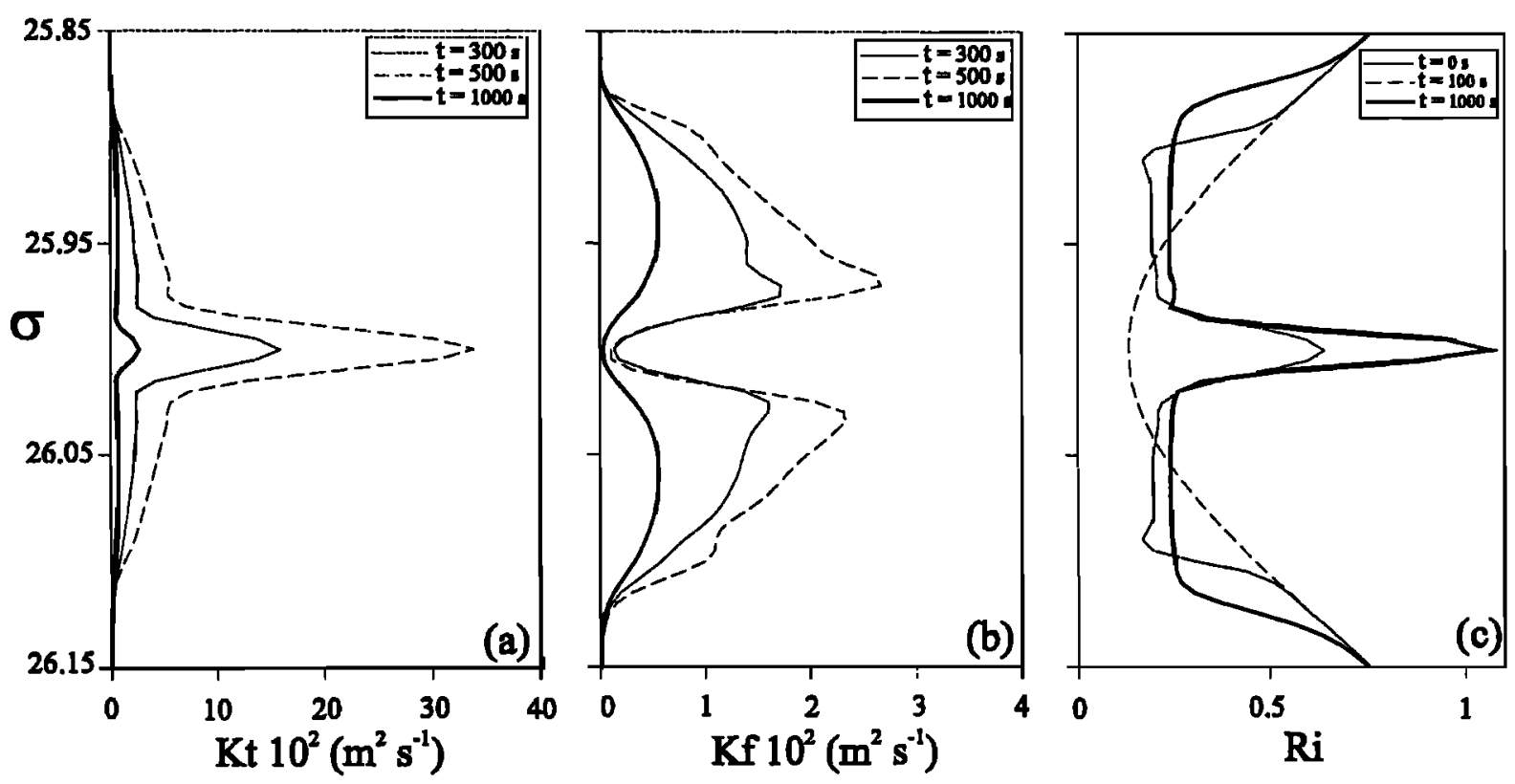

Figure 8. Case with $\tau=N^{-1}$, showing the temporal evolution of (a) actual density diffusivity, (b) instantaneous forcing density diffusivity, and (c) local gradient Richardson number.

ures 9 and 10. We have made other tests, such as letting $\partial v / \partial \rho$ decrease with time, and similar results have been obtained. The conclusion is that as long as $\partial v / \partial \rho$ remains of the same order, the level of turbulence (and hence $K_{f}$ ) is substantially unchanged.

Despite the conceptual justifications for a constant diapycnal shear and the relatively low sensitivity of the model to changes in $\partial v / \partial \rho$, it is clear that the lack of dynamics is a major limitation of our model. However, we believe that for geophysical flows our simple model probably reproduces the main features, leading to the evolution from continuous stratification into mixed layers. This statement is further supported by the following consideration. In our model the temporal memory of turbulence is related to the inverse buoyancy frequency. This poses a lower limit to the time required for changes in the vertical density flux, independent of faster changes in the diapycnal shear. For geostrophically balanced flows, however, this limit should actually be much larger. The reason is that rapid changes in the isopycnal slopes, such as those caused by localized mixing, are not immediately followed by a change in the geostrophic diapycnal shear. This delay is related to the relatively large characteristic timescale of geostrophic flows, much larger than the inverse buoyancy frequency, and it should be indicative of the actual time required for substantial changes in the diapycnal shear to take place.

We have argued that shear-induced, Kelvin-Helmholtz type, instabilities are the likely physical mechanism responsible for mixing in the reported numerical calculations. This conclusion is based on the fact that the eddy density flux is parameterized in terms of the gradient Richardson number, as expected for these instabilities, and that there is experimental and field data that corroborates their presence in the development of mixed layers. It is possible, however, that our numerical simulations may also represent the generation of mixed layers by other physical mechanisms. $D e$ Silva and Fernando [1992], for example, have reported laboratory experiments in which a grid oscillating in an initial linearly stratified fluid produces a well-mixed turbulent patch. In their experiments, with no mean shear, the source of turbulence is clearly not related to Kelvin-Helmholtz instabilities. The main difficulty with this and other possible sources of instability is to identify how the physical mechanism operates and how the vertical density flux should be parameterized. This is clearly related to De Silva and Fernando's [1992] warning that "in the oceanic context the exact nature and strength of turbulent sources are not known and the extrapolation of the laboratory results to oceanic cases should be done with caution." For this reason, we believe that it is particularly important not to separate numerical solutions from physical reasoning.

\section{Conclusions}

We have presented a simple process-oriented model that studies the formation of mixed layers in stratified geophysical flows. The model is based on the idea that well-stratified regions with high diapycnal shear are dynamically unstable and Kelvin-Helmholtz type instabilities develop. These instabilities cause the stratified region to mix and adjacent interfaces to be formed. For this mechanism to operate the vertical density diffusivity must fulfill two conditions. First, the diffusivity must monotonically increase with decreasing gradient Richardson numbers, and second, it must have a temporal memory of turbulence. 

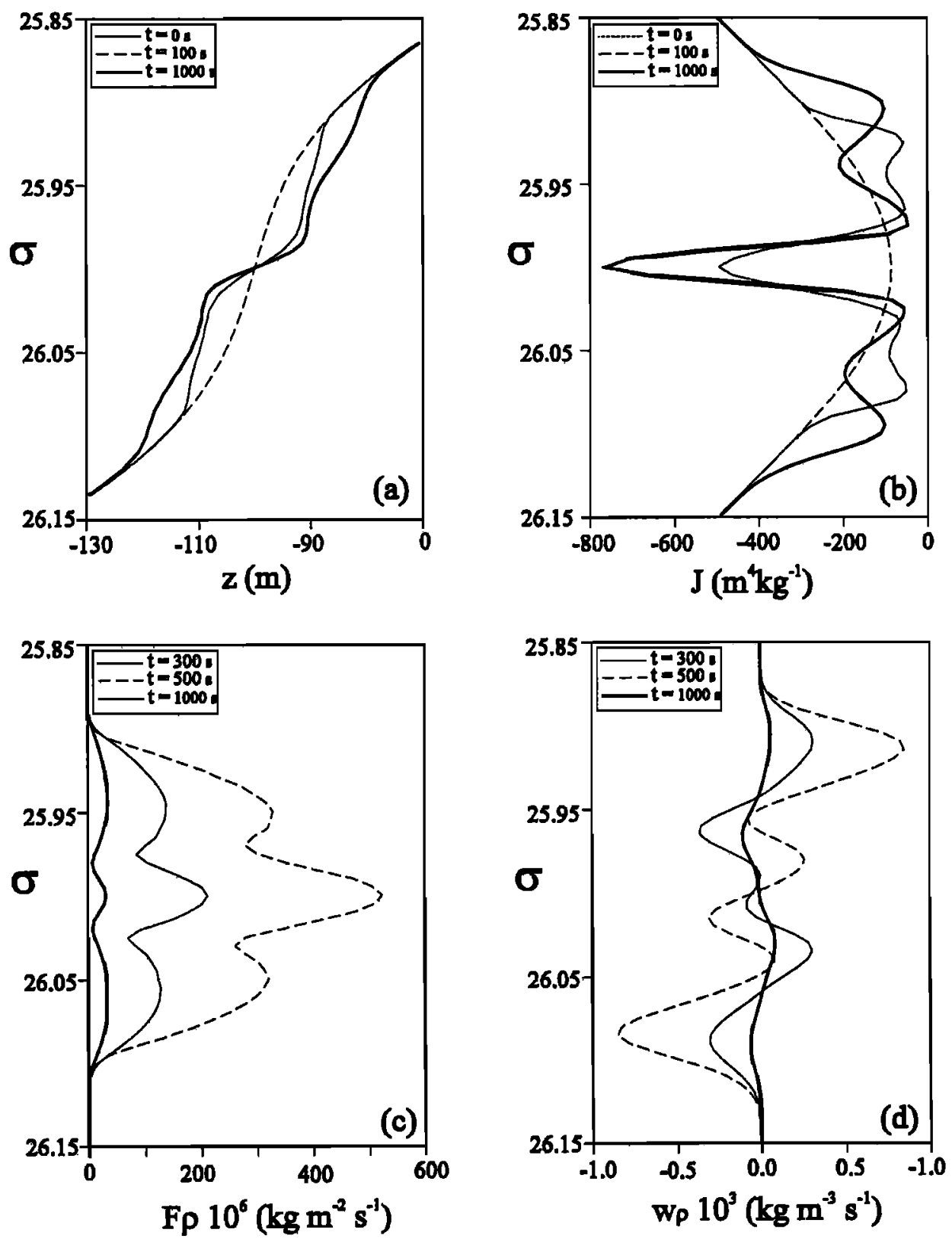

Figure 9. Case with $\tau=N^{-1}$ and a running filter applied to $R i$, showing the temporal evolution of the density distribution for (a) depth, (b) Jacobian, (c) vertical density flux, and (d) density tendency.

Our numerical calculations show that an initially highly stratified but unstable region is merely diffusive if turbulence has no memory. If we allow a finite temporal adjustment for turbulence, however, the stratified region becomes well mixed and sharp interfaces are generated above and below. If we further take into account the minimum vertical scale for Kelvin-Helmholtz instabilities, then the staircase becomes clearer, with the adjacent stratified regions showing some breaking into new mixed layers.

The numerical results shown correspond to constant diapycnal shear, but several tests indicate that the results are rather robust and depend little on temporal changes of the diapycnal shear. Further studies are re- quired to incorporate more realistic dynamics, including vertical mixing of momentum and the evolution of geostrophic shear flow in frontogenetical systems. Additional efforts are also required to obtain both laboratory and field data in order to properly validate the proposed mechanism.

The mechanism we have presented clearly differs from Phillips' in two main respects. First, the dynamics of the instability process is different: shear-induced mixing rather than mixing generated through oscillating rods. A consequence is that for Phillips, the vertical density diffusivity has a maximum as a function of some properly defined local Richardson number, which is not the local gradient Richardson number Ri. However, the 

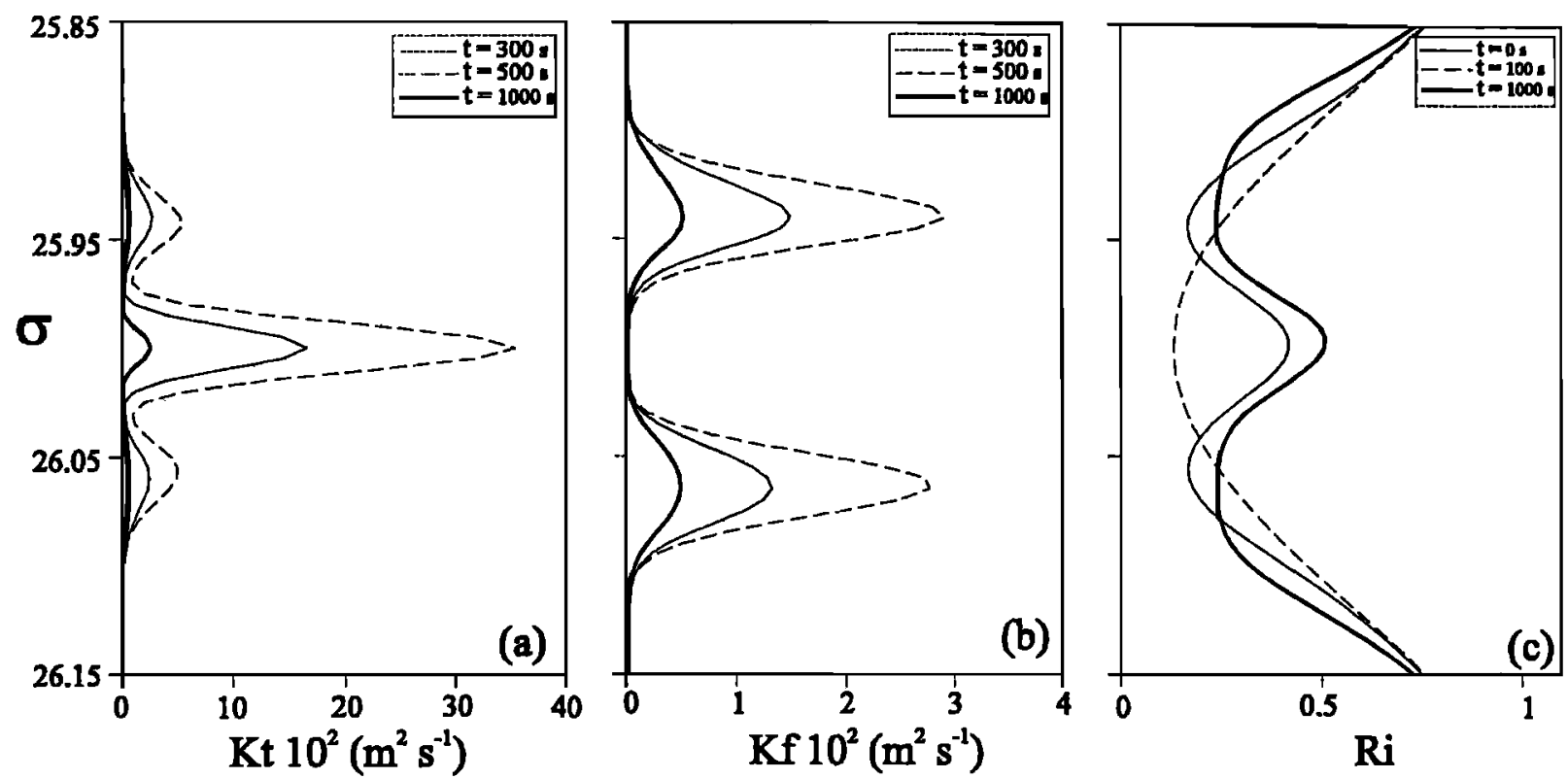

Figure 10. Case with $\tau=N^{-1}$ and a running filter applied to $R i$, showing the temporal evolution of (a) actual density diffusivity, (b) instantaneous forcing density diffusivity, and (c) local gradient Richardson number.

vertical density diffusivity in the proposed mechanism monotonically increases with decreasing $R i$. Second, the kinematics of mixing is totally opposite: a highly stratified region splitting into a mixed region and two adjacent interfaces rather than a growing perturbation in the depth-density profile.

Acknowledgments. This paper had its original motivation from Gabe Csanady's emphasis on thinking in terms of isopycnic coordinates. We wish to thank him for suggesting this approach as well as for his contagious enthusiasm on physical reasoning. We are also grateful to one anonymous reviewer for a number of useful comments. This work has been supported by the Spanish government through project FRENTES (CICYT grant AMB95-0731) and by the European Union through project CANIGO (MAST grant MAS3CT96-0060).

\section{References}

Arístegui, J., P. Sangrà, S. Hernández-León, M. Cantón, A. Hernández-Guerra, and J. L. Kerling, Island-induced eddies in the Canary Islands, Deep Sea Res., 41, 1509$1525,1994$.

Bane, J. M., D. A. Brooks, and K. R. Lorenson, Synoptic observations of the three-dimensional structure and propagation of Gulf Stream meanders along the Carolina continental margin, J. Geophys. Res., 86, 6411-6425, 1981.

Barenblatt, G. I., M. Bertsch, R. dal Passo, V. M. Prostokishin, and M. Ughi, A mathematical model of turbulent heat and mass transfer in stably stratified shear flow, J. Fluid Mech., 253, 341-358, 1993.

Browning, K. A., and C. D. Watkins, Observations of clear air turbulence by high power radar, Nature, 227, 260-263, 1970.

Cisneros-Aguirre, J., J. L. Pelegrí, and P. Sangrà, Experiments on layer formation in stratified shear flows, paper presented at Meeting on Mixing in Geophysical Flows
II, Universitat Politécnica de Catalunya, Barcelona, April 1997.

Csanady, G. T., Turbulent Diffusion in the Environment, 248 pp., D. Reidel, Norwell, Mass., 1973.

Csanady, G. T., Intermittent "full" upwelling in Lake Ontario, J. Geophys. Res., 82, 397-419, 1977.

Csanady, G. T., Shelf circulation cells, Philos. Trans. R. Soc. London, Ser. A, 302, 515-530, 1981.

Csanady, G. T., On the structure of transient upwelling events, J. Phys. Oceanogr., 12, 84-96, 1982.

Csanady, G. T., Radiation of topographic waves from Gulf Stream meanders, Continental Shelf Res., 8, 673-686, 1988.

Csanady, G. T., Energy dissipation and upwelling in a western boundary current, J. Phys. Oceanogr., 19, 462-473, 1989.

Csanady, G. T., Mixing in coastal regions, in The Sea: Ocean Engineering Science, vol. 9, edited by B. Le Mehau te and D. M. Hanes, pp. 593-629, John Wiley, New York, 1990.

Csanady, G. T., and J. L. Pelegrí, Vorticity balance of boundary currents, J. Mar. Res., 53, 171-187, 1995.

De Silva, L. P. D., and H. J. S. Fernando, Some aspects of mixing in a stratified turbulent patch, J. Fluıd Mech., 240, 601-625, 1992.

Fernando, H. J. S., Turbulent mixing in stratified fluids, Annu. Rev. Fluid Mech., 23, 455-493, 1991.

Fernando, H. J. S., Experiments on some stratified and rotating flows of geophysical relevance, paper presented at the Meeting on Mixing in Geophysical Flows II, Universitat Politécnica de Catalunya, Barcelona, April 1997.

Gregg, M. C., Diapycnal mixing in the thermocline: A review, J. Geophys. Res., 92, 5249-5286, 1987.

Holt, S. E., J. R. Koseff, and J. H. Ferziger, A numerical study of the evolution and structure of homogeneous stably stratified sheared turbulence, J. Fluid. Mech., 237, 499-539, 1992.

Howard, L. N., Note on a paper by John W. Miles, J. Fluid Mech., 95, 509-512, 1961. 
Ivey, G. N., and J. Imberger, On the nature of turbulence in stratified fluid, Part I, The energetics of mixing, J. Phys. Oceanogr., 21, 650-658, 1991.

Kranenburg, C., On gradient transport turbulence models for stably stratified shear flows, Dyn. Atmos. Oceans, 23, 205-215, 1996.

Lee, S., and G. T. Csanady, Instability waves in the Gulf Stream front and its thermocline layer, J. Mar. Res., 52, 837-863, 1994.

Linden, P. F., Mixing in stratified fluids, Geophys. Astrophys. Fluid Dyn., 13, 3-23, 1979.

Linden, P. F., Mixing across a density interface produced by grid turbulence, J. Fluid Mech., 100, 691-703, 1980.

McDougall, T. J., and Y. You, Implications of the nonlinear equation of state for upwelling in the ocean interior, J. Geophys. Res., 95, 13,263-13,276, 1990.

Miles, J. W., On the stability of heterogeneous shear flows, J. Fluid Mech., 10, 496-508, 1961.

Miller, J. L., and D. L. Evans, Density and velocity enhancement in oceanic eddies, J. Geophys. Res., 90, 4793-4806, 1985.

Munk, W. H., and E. R. Anderson, Notes on a theory of the thermocline, J. Mar. Res., 7, 276-295, 1948.

Pacanowski, R. C., and S. G. H. Philander, Parameterization of vertical mixing in numerical models of tropical oceans, J. Phys. Oceanogr., 11, 1443-1451, 1981

Park, Y., J. A. Whitehead, and A. Gnanadeskian, Turbulent mixing in stratified fluids: Layer formation and energetics, J. Fluid Mech., 279, 279-311, 1994.

Pelegrí, J. L., and G. T. Csanady, Nutrient transport and mixing in the Gulf Stream, J. Geophys. Res., 96, 25772583, 1991.

Pelegrí, J. L., and G. T. Csanady, Diapycnal mixing in western boundary currents, J. Geophys. Res., 99, 18,27518,304, 1994.

Pelegrí, J. L., G. T. Csanady, and A. Martins, The North Atlantic nutrient stream, $J$. Oceanogr., 52, 275-299, 1996.

Peters, H., M. C. Gregg, and J. M. Toole, On the parameterization of equatorial turbulence, J. Geophys. Res., 93, 1199-1218, 1988.

Phillips, O. M., Turbulence in a strongly stratified fluid - Is it unstable?, Deep Sea Res., 19, 79-81, 1972.
Pollard, R. T., P. B. Rhines, and R. O. R. Y. Thompson, The deepening of the wind-mixed layer, Geophys. Fluid Dyn., 4, 381-404, 1973.

Posmentier, E. S., The generation of salinity finestructure by vertical diffusion, J. Phys. Oceanogr., 7, 298-300, 1977.

Rossby, C. G., Dynamics of steady ocean currents in the light of experimental fluid mechanics, Papers Phys. Oceanogr. Meteorol., 5, 1-43, 1936.

Ruddick, B. R., T. J. McDougall, and J. S. Turner, The formation of layers in a uniformly stirred density gradient, Deep Sea Res., 36, 597-609, 1989.

Shapiro, R., Smoothing, filtering, and boundary effects, Rev. Geophys., 8, 359-387, 1970.

Simpson, J. H., The shelf-sea fronts: Implications of their existence and behaviour, Philos. Trans. R. Soc. London, Ser. A, 302, 531-546, 1981.

Thorpe, S. A., Experiments on the instability of stratified shear flow: miscible fluids, $J$. Fluid Mech., 46, 299-319, 1971.

Thorpe, S. A., Transitional phenomena and the development of turbulence in stratified fluids: A review, J. Geophys. Res., 92, 5231-5248, 1987.

Turner, J. S., Buoyancy Effects in Fluids, 367 pp., Cambridge Univ. Press, New York, 1973.

Turner, J. S., Turbulent entrainment: The development of the entrainment assumption, and its application to geophysical flows, J. Fluid Mech., 173, 431-471, 1986.

Woods, J. D., Wave-induced shear instability in the summer thermocline, J. Fluid Mech., 32, 791-800, 1968.

Woods, J. D., and R. L. Wiley, Billow turbulence and ocean microstructure, Deep Sea Res., 19, 87-121, 1972.

J. L. Pelegrí and P. Sangrà, Facultad de Ciencias del Mar, Campus Universitario de Tafira, Universidad de Las Palmas de Gran Canaria, 35017 Las Palmas de Gran Canaria, Spain. (e-mail: joseluis.pelegri@fisica.ulpgc.es; pablo.sangra@fisica.ulpgc.es)

(Received August 29, 1996; revised January 21, 1998; accepted January 30, 1998.) 